\title{
Teleparallel spin connection
}

\author{
V. C. de Andrade \\ Département d'Astrophysique Relativiste et de Cosmologie, Centre National de la Recherche Scientific (UMR 8629), \\ Observatoire de Paris, 92195 Meudon Cedex, France \\ L. C. T. Guillen and J. G. Pereira \\ Instituto de Física Teórica, Universidade Estadual Paulista, Rua Pamplona 145, 01405-900 São Paulo SP, Brazil
}

(Received 8 December 2000; published 4 June 2001)

\begin{abstract}
A new expression for the spin connection of teleparallel gravity is proposed, given by minus the contorsion tensor plus a zero connection. The corresponding minimal coupling is covariant under local Lorentz transformation, and equivalent to the minimal coupling prescription of general relativity. With this coupling prescription, therefore, teleparallel gravity turns out to be fully equivalent to general relativity, even in the presence of spinor fields.
\end{abstract}

DOI: 10.1103/PhysRevD.64.027502

PACS number(s): 04.20.Cv, 04.50. $+\mathrm{h}$

In the absence of spinor fields, the equivalence of teleparallel gravity [1] with general relativity is a well established subject [2]. In the presence of spinor fields, however, it is widely believed that this equivalence is spoiled [3]. The reason for this is that, in teleparallel gravity, the spin connection is believed to vanish. The basic purpose of this Brief Report is to show that this conclusion is not appropriate, and that it comes from a particular choice of the teleparallel spin connection. A new expression for the spin connection is then proposed, according to which teleparallel gravity becomes much more consistent and fully equivalent to general relativity, even in the presence of spinor fields.

We use the Greek alphabet $(\mu, \nu, \rho, \ldots=0,1,2,3)$ to denote indices related to spacetime (base space), and the Latin alphabet $(a, b, c, \ldots=0,1,2,3)$ to denote indices related to the tangent space (fiber), assumed to be a Minkowski spacetime. The spin connection $\stackrel{\circ}{\mu}_{\mu}$ can thus be written as

$$
\stackrel{\circ}{A}_{\mu}=\frac{1}{2} \stackrel{\circ}{A}^{a b}{ }_{\mu} S_{a b},
$$

where $S_{a b}$ is an element of the Lorentz Lie algebra written in an appropriate representation.

In general relativity, the spin connection is [4]

$$
\stackrel{\circ}{A}_{b \nu}^{a}=h_{\rho}^{a} \stackrel{\circ}{\Gamma}^{\rho}{ }_{\mu \nu} h_{b}{ }^{\mu}+h^{a}{ }_{\rho} \partial_{\nu} h_{b}{ }^{\rho},
$$

where $h_{\mu}^{a}$ is a tetrad field, and $\stackrel{\circ}{\Gamma}_{\mu \nu}$ is the Levi-Civita connection. In order to obtain the teleparallel spin connection, inspired by the definition (2), it is usual to start by making the following attempt:

$$
A_{b \nu}^{a}=h_{\rho}^{a} \Gamma_{\mu \nu}^{\rho} h_{b}{ }^{\mu}+h^{a}{ }_{\rho} \partial_{\nu} h_{b}{ }^{\rho},
$$

with $\Gamma_{\mu \nu}^{\rho}$ the Weitzenböck connection. However, as a consequence of the absolute parallelism condition [2], we have $A_{b \nu}^{a}=0$. This does not mean that in teleparallel gravity the spin connection vanishes. It only means that we have not made an appropriate guess.

Let us then adopt a different procedure to look for the teleparallel spin connection. Our basic guideline will be to find a coupling prescription that results equivalent to the coupling prescription of general relativity. This can be achieved by taking the relation between the Levi-Civita and the Weitzenböck connections [5],

$$
\stackrel{\circ}{\Gamma}_{\mu \nu}^{\rho}=-K_{\mu \nu}^{\rho}+\Gamma_{\mu \nu}^{\rho}
$$

with $K^{\rho}{ }_{\mu \nu}$ the contorsion tensor, and rewriting it in the tetrad basis. The result is

$$
\stackrel{\circ}{A}_{b \mu}^{a}=-K_{b \mu}^{a}+0,
$$

where we have already used the fact that $A^{a}{ }_{b \mu}=0$. Notice that the zero connection appearing in Eq. (5) is crucial in the sense that it is the responsible for making the right-hand side a true connection. Therefore, based on these considerations, we can say that the teleparallel spin connection $\omega_{b \mu}^{a}$ is given by minus the contorsion tensor plus a zero connection:

$$
\omega_{b \mu}^{a}=-K_{b \mu}^{a}+0 .
$$

Accordingly, the teleparallel Fock-Ivanenko derivative operator is to be written in the form

$$
\mathcal{D}_{\mu}=\partial_{\mu}-\frac{i}{2} \omega^{a}{ }_{b \mu} S_{a}{ }^{b} .
$$

It is important to remark that, differently from $\stackrel{\circ}{A}^{a}{ }_{b \mu}$ which depends on the Levi-Civita connection only, $\omega^{a}{ }_{b \mu}$ depends on the Weitzenböck connection only.

The covariant derivative (7) presents all necessary properties to be considered as yielding the fundamental coupling prescription in teleparallel gravity. It transforms covariantly under local Lorentz transformations, it reduces to the teleparallel version of the Levi-Civita covariant derivative when applied to tensor fields [6], and it turns out to be completely equivalent to the usual minimal coupling prescription of general relativity, even in the presence of spinor fields. Therefore, we can conclude that $\omega_{b \mu}^{a}$ presents all 
necessary properties to be considered as the spin connection of teleparallel gravity. The consistency of this result can also be checked by noticing that, under a local Lorentz transformation with parameters $\epsilon_{b}^{a}$, the spin connection $\omega^{a}{ }_{b \mu}$ changes according to

$$
\delta \omega^{a}{ }_{b \mu}=-\mathcal{D}{ }_{\mu} \epsilon_{b}^{a},
$$

which is the standard connection transformation of gauge theories [7].

The authors are indebted to R. Aldrovandi and C. M. Zhang for valuable comments. They also acknowledge FAPESP-Brazil, CAPES-Brazil, and CNPq-Brazil for financial support.
[1] For a general review on the gauge approach to gravity, see F. W. Hehl, J. D. McCrea, E. W. Mielke, and Y. Neèmann, Phys. Rep. 258, 1 (1995).

[2] V. C. de Andrade and J. G. Pereira, Phys. Rev. D 56, 4689 (1997).

[3] K. Hayashi and T. Shirafuji, Phys. Rev. D 19, 3524 (1979).

[4] P. A. M. Dirac, in Planck Festscrift, edited by W. Frank (Deut- scher Verlag der Wissenschaften, Berlin, 1958).

[5] V. C. de Andrade, L. C. T. Guillen, and J. G. Pereira, Phys. Rev. Lett. 84, 4533 (2000).

[6] V. C. de Andrade and J. G. Pereira, Int. J. Mod. Phys. D 8, 141 (1999).

[7] See, for example, P. Ramond, Field Theory: A Modern Primer (Benjamin/Cummings, Reading, 1981). 